\title{
Two-dimensional reward evaluation in mice
}

\author{
Vladislav Nachev $^{1}\left[\right.$ Marion Rivalan $^{1,2} \cdot$ York Winter $^{1,2}$
}

Received: 3 November 2020 / Revised: 18 January 2021 / Accepted: 20 January 2021 / Published online: 15 March 2021 (c) The Author(s) 2021

\begin{abstract}
When choosing among multi-attribute options, integrating the full information may be computationally costly and timeconsuming. So-called non-compensatory decision rules only rely on partial information, for example when a difference on a single attribute overrides all others. Such rules may be ecologically more advantageous, despite being economically suboptimal. Here, we present a study that investigates to what extent animals rely on integrative rules (using the full information) versus non-compensatory rules when choosing where to forage. Groups of mice were trained to obtain water from dispensers varying along two reward dimensions: volume and probability. The mice's choices over the course of the experiment suggested an initial reliance on integrative rules, later displaced by a sequential rule, in which volume was evaluated before probability. Our results also demonstrate that while the evaluation of probability differences may depend on the reward volumes, the evaluation of volume differences is seemingly unaffected by the reward probabilities.
\end{abstract}

Keywords Multi-attribute choice $\cdot$ Non-compensatory decision rules $\cdot$ Economic decision-making $\cdot$ Home cage testing $\cdot$ Mice

\section{Introduction}

Animals confronted with options that differ on a single attribute generally make economically rational choices consistent with gain maximization (Monteiro et al. 2013; Rivalan et al. 2017). In multi-attribute choice (Pitz and Sachs 1984; Jansen et al. 2012; Hunt et al. 2014), however, where reward attributes must be weighed against each other (price vs. quality, risk vs. pay-off, etc.), consistent deviations from economical rationality have been described in humans (Tversky and Kahneman 1974; Rieskamp et al. 2006; Katsikopoulos and Gigerenzer 2008) and non-human animals (Shafir et al. 2002; Bateson et al. 2003; Schuck-Paim et al. 2004; Scarpi 2011; Nachev and Winter 2012; Nachev et al. 2017; Constantinople et al. 2019). Some deviations from gain maximization can be accounted for by considering the ecological circumstances of an animal, which may confer fitness benefits to seemingly irrational choices (Kacelnik 2006; Houston et al. 2007; Trimmer 2013; McNamara et al. 2014).

Vladislav Nachev

vladislav.nachev@gmail.com

1 Institute of Biology, Humboldt University, Philippstr. 13, 10099 Berlin, Germany

2 Charité University Medicine, Berlin, Germany
An animal foraging in its natural environment mostly encounters food items that differ on multiple attributes, but only some of those attributes affect the long-term gains. We refer to those attributes as reward dimensions. In multidimensional choice, the decision task is considerably simplified if differences that are (nearly) equal are not evaluated but ignored (Tversky 1969; Pitz and Sachs 1984; Shafir 1994; Shafir and Yehonatan 2014). For example, an animal might only consider the one reward dimension (e.g., prey size) that most strongly affects the long-term gains. Such decision processes in which one reward dimension overrides the others have been described as non-compensatory (Pitz and Sachs 1984; Reid et al. 2015) and can potentially increase speed of decision and decrease computation costs at the expense of accuracy. Attributes can be considered sequentially, for example ranked by salience, until a sufficient difference is detected on one attribute, so that a decision can be reached (Brandstätter et al. 2006; Jansen et al. 2012). In compensatory decision-making (Pitz and Sachs 1984; Reid et al. 2015) on the other hand, choice is affected by multiple attributes that are integrated into a common decision currency (utility) (Levy and Glimcher 2012). A fully integrative approach that makes use of all the available information (also referred to as absolute reward evaluation Tversky 
1969; Shafir 1994; Shafir and Yehonatan 2014) is equivalent to gain maximization. For example, if options differ along the reward dimensions of amount and probability of obtaining this amount, maximizing the gain is ensured by selecting the option with the highest expected value, which is the product of the amount and probability. Even in two-dimensional reward evaluation, a range of strategies are possible, from sequential and other non-compensatory rules, up to full integration.

When studying animal decision-making, preferences are measured over many choices, especially when options differ in reward probability. Although a rational subject should exclusively select the most profitable option, animals can persist in choosing less profitable options even after long training, usually at some low frequency (Kacelnik 1984). The partial preference observed in choice experiments can be explained by profitability matching (Kacelnik 1984), which states that animals proportionally allocate their effort depending on the relative pay-off of the options.

Scalar utility theory (SUT: Kacelnik and Brito e Abreu 1998; Marsh and Kacelnik 2002) is a framework that proposes a proximate mechanism that accounts for partial preferences in the context of reward amount and reward variability (Rosenström et al. 2016). Based on findings in psychophysics, SUT postulates that cognitive representations of stimuli exhibit a scalar property, i.e., they have error distributions that are normal with a mean equal to the magnitude of the stimulus and a standard deviation that is proportional to the mean. In other words, SUT states that the memory traces of perceived or expected outcomes of choices are subject to Weber's law (Akre and Johnsen 2014) and that rewards are evaluated proportionally rather than linearly (Marsh and Kacelnik 2002; Rosenström et al. 2016). Therefore, according to SUT, choice is modelled by sampling from the internal representations of the choice options and selecting the most favorable sample. This allows for making quantitative predictions about the strength of preferences from the contrasts between options.

In previous experiments, we have demonstrated that proportional processing can be used to predict the choice behavior of animals when options vary along a single dimension (Nachev et al. 2013; Rivalan et al. 2017). In the present study, we extend the application of proportional processing and SUT to two-dimensional choice tasks with the aim to test whether (contradictory) information from two reward dimensions generates choices more consistent with integrative or non-compensatory decision rules. We used a combination of behavioral studies of mice and a decision-making model based on SUT.

\section{Animals, methods, and materials}

\section{Animals}

The experiments were conducted with three cohorts of C57BL/6NCrl female mice (Charles River, Sulzfeld, Germany, total $n=30$ ). Mice were 5 weeks old on arrival. The mice from each cohort were housed together, before and during the experiments. They were marked with unique radiofrequency identification tags (RFID: $12 \times 2.1 \mathrm{~mm}, 125 \mathrm{kHz}$, Sokymat, Rastede, Germany) under the skin in the scruff of the neck and also earmarked at age 6 weeks. At age 7 weeks, mice were transferred to the automated group home cage for the main experiment. Pellet chow (V1535, maintenance food, ssniff, Soest, Germany) was always accessible from a trough in the cage lid. Water was available from the operant modules of the automated group cage, depending on individual reward schedules. Light conditions in the experiments were $12: 12 \mathrm{LD}$ and climatic conditions were $23 \pm 2,{ }^{\circ} \mathrm{C}$ and $50-70 \%$ humidity.

\section{Ethics statement}

The experimental procedures were aimed at maximizing animal welfare. During experiments, mice remained undisturbed in their home cage. Data collection was automated, with animals voluntarily visiting water dispensers to drink. The water intake and health of the mice was monitored daily. Due to the observational nature of the study, animals were free from damage, pain, and suffering. The animals were not sacrificed at the end of the study, which was performed under the supervision and with the approval of the animal welfare officer heading the animal welfare committee at Humboldt University. Experiments followed national regulations in accordance with the European Communities Council Directive 10/63/EU.

\section{Cage and dispenser system}

We used two automated home cages $(612 \times 435 \times 216 \mathrm{~mm}$, P2000, Tecniplast, Buggugiate, Italy) with woodchip bedding (AB 6, AsBe-wood, Gransee, Germany), and enriched with two gray PVC tubes and paper towels as nesting material. The cage was outfitted with four computer-controlled liquid dispensers. The experimental set-up of cage 1 is described in detail in Rivalan et al. (2017). Briefly, mice were detected at the dispensers via infrared beam-break sensors and RFID sensors. Water delivery at each dispenser could be controlled, so that it could be restricted or dispensed at different amounts on an individual basis. Mice were therefore rewarded with droplets of water from the 
dispenser spout that they could remove by licking. We changed cage bedding and weighed all animals on a weekly basis, always during the light phase and at least an hour before the start of the testing session. Data were recorded and stored automatically on a laptop computer using PhenoSoft Control software (PhenoSys, Germany). Timestamped nose poke events and amounts of water delivered were recorded for each dispenser, with the corresponding mouse identity.

The second automated group cage (cage 2) was made for the purposes of this study and was nearly identical to cage 1 . The crucial modification was that the stepping-motor syringe pump was replaced with a model that used disposable plastic 25-mL syringes (cage 2) instead of gas-tight Hamilton glass syringes (Series 1025, cage 1). Thus, the pumping systems in the two cages differed in the smallest reward that could be delivered and in the precision of reward delivery (mean \pm SD: $0.33 \pm 0.03 \mu \mathrm{L} \mathrm{step}^{-1}$ in cage 1 vs. $1.56 \pm 0.24 \mu \mathrm{L} \mathrm{step}^{-1}$ in cage 2). The precision of each pump was estimated by manually triggering reward visits at different preset pump steps (17 and 42 in cage 1, 3 and 12 in cage 2 ) and collecting the expelled liquid in a graduated glass pipette placed horizontally next to the cage. Each dispenser was measured by the same trained experimenter at least 20 times for each pump step value.

\section{Experimental schedule}

The general experimental procedure was as described before (Rivalan et al. 2017). The water dispensers were only active during an 18-h-long drinking session each day that began with the onset of the dark phase and ended $6 \mathrm{~h}$ after the end of the dark phase. The reward properties (volume and probability) were dependent on the experimental condition. Rewards were drawn from fixed pseudo-random repeating sequences. These sequences were: 11101111101101111110 for $80 \%, 11011101110101101110$ for $70 \%$, 10110101101001001010 for $50 \%, 10010100100001001000$ for $30 \%$, and 10001000010001000000 for $20 \%$, where 1 is a rewarded nose poke and 0 is an unrewarded nose poke.

Although individual mice shared the same dispensers inside the same cage, they were not necessarily in the same experimental phase during training or experimental condition in the main experimental phase. The three cohorts (1-3 in chronological order) were tested consecutively, with cohort 2 housed in cage 2 and the other cohorts housed in cage 1 . If after any drinking session during any experimental phase a mouse drank less than $1 \mathrm{~mL}$ of water, we placed two water bottles in the automated cage, gently awakened all mice, and allowed them to drink freely until they voluntarily stopped. An overview of the training and experimental phases is given in Fig. 1a.

\section{Exploration phase}

At the beginning of this phase, there were ten mice in each cohort, except for cohort 2 , in which one mouse was excluded due to the loss of the RFID tag after implantation (the mouse was in good health condition). The mice were transferred to the automated cages $1-2 \mathrm{~h}$ before the first drinking session of the exploration phase. The purpose of this phase was to let mice accustom to the cage and learn to use the dispensers to obtain water. Therefore, each nose poke at any dispenser was rewarded with a constant volume of $20 \mu \mathrm{L}$. The criterion for advancing to the following training phase was consuming more than $1 \mathrm{~mL}$ in a single drinking session. Mice that did not reach the criterion remained in the exploration phase until they either advanced to the following phase or were excluded from the experiment $(n=1$ mouse in cohort 2).

\section{Training phase}

In this phase, the reward volume was reduced to $10 \mu \mathrm{L}$ and the reward probability was reduced to 0.3 at all dispensers. These reward values ensured that mice remained motivated to make several hundred visits per drinking session. Associative learning is also enhanced by the unpredictability of the expected reinforcer (Maddux et al. 2007). The training phase was repeated for 1-2 days until at least eight mice fulfilled the criterion of consuming more than $1 \mathrm{~mL}$ of water in one drinking session. The purpose of the training phase was to introduce mice to the reward dimensions (volume and probability) that would be used in the following discrimination experiments. In cohorts 1 and 2, mice were excluded from the experiment if they did not reach the criterion in 2 days, or, alternatively, if more than eight mice had reached the criterion, mice were excluded at random to ensure a balanced number of mice per dispenser. These mice were returned to regular housing.

\section{Noise habituation}

We introduced a noise habituation phase for the mice in cohort 3 , because after 2 days, only six of them had advanced to the training phase (Fig. 1a). The unusually low number of visits made by mice that did not pass the exploration phase suggested that the noise produced by the pumping systems might scare naive, shy mice away from the dispensers. To ensure that all mice were successfully trained, we designed the noise habituation, so that rewards at all four dispensers were delivered at regular intervals ( 7 $\mu \mathrm{L}$ every minute), regardless of the behavior of the mice. After 2 days, all mice had made at least 200 nose pokes and the cohort then continued with either exploration or training. Two days later, all mice successfully completed the training 
Fig. 1 Experimental conditions and schedules. a Experimental schedule with all phases. The number of days is given in parentheses. Mice began with an exploration phase, followed by a training phase. Before every experiment (Exp. 1-4), there was another training phase for 1 day. Between experiments 3 and 4, there was a 4-day break with water from a bottle. Phases shown with dashed lines were only present in the schedule for cohort 3 , because four mice had difficulties advancing beyond the exploration phase. b Behavioral task in four conditions (BPLV, BVHP, C, and I) of experiment 1. Mice were free to nosepoke in all four corners, two of which (shown in blue) were rewarding for the example mouse, with the reward properties shown above or below the reward corners. For clarity, only one example mouse of the eight mice is shown, with other mice experiencing different conditions at different dispensers. $B P L V$ baseline for probability at low volume, $B V H P$ baseline for volume at high probability, $C$ congruent condition, $I$ incongruent condition (colour figure online)

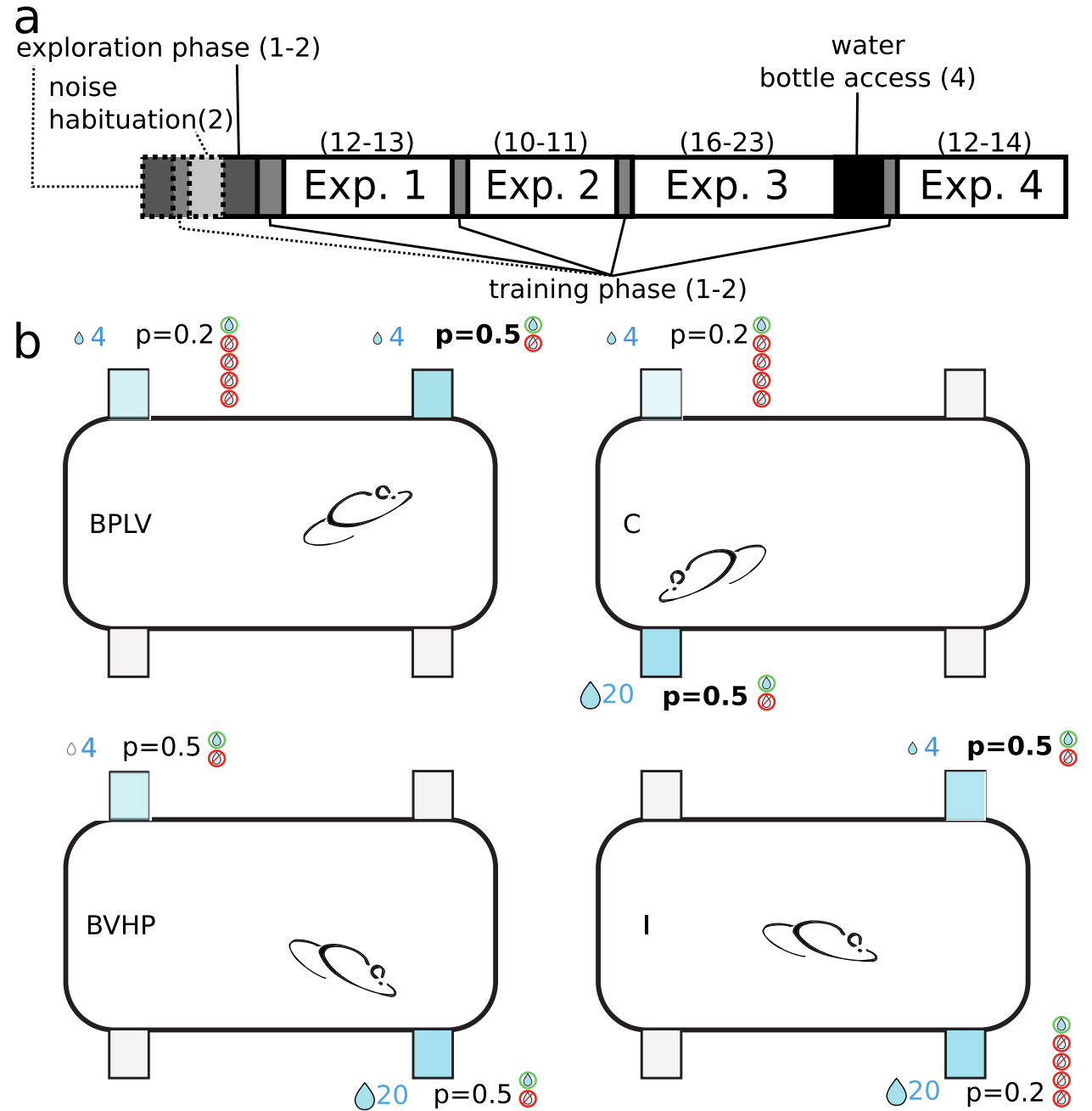

phase and two mice were randomly selected for removal from the experiment, bringing the number of mice to eight. We therefore updated our training procedure to always begin with noise habituation, followed by the exploration phase and the training phase.

\section{General procedure in the main experiments}

After eight mice had successfully passed the training phase, they proceeded with experiment 1 from the main experiments (1-4). In all of the main experiments, mice had a choice between four dispensers, where two were not rewarding and the other two gave rewards with volumes and probabilities that depended on the experimental condition (Figs. 1b, 2). In most conditions, one of the rewarding dispensers (high-profitability dispenser) was more profitable than the other (low-profitability dispenser). The sequence of conditions was randomized for each individual, so that any given mouse was usually experiencing a different experimental condition than all other mice. On any given day, two of the dispensers were rewarding for four mice and the other two were rewarding for the other four mice. Within each group of four, each pair of mice shared the same high- and low-profitability dispensers, which were spatially inverted between pairs of mice. This pairing was done to increase the throughput of the experiments, while controlling for potential social learning effects and distributing mice evenly over the dispensers to minimize crowding effects.

The behavioral measure of interest was the relative visitation rate to the high-profitability dispenser that could develop in one drinking session. Choice behavior in sequential testing with multiple conditions can be influenced by the previous conditions and by side bias. We aimed to mitigate the sequential effects through randomization, and the side bias through spatially reversing the choice options. As a control for positional biases, each condition was followed by a reversal on the next day, so that the high- and low-profitability dispensers were spatially inverted for all mice, whereas the two non-rewarding dispensers remained unchanged. Reversal was followed by the next experimental condition, with pseudo-random distribution of the dispensers among the pairs of mice 


\begin{tabular}{|c|c|c|c|c|c|}
\hline experiment & condition & $\begin{array}{l}\text { volume } \\
(\mathrm{A} \mid \mathrm{B})\end{array}$ & $\begin{array}{c}\text { probabilities } \\
(\mathrm{A} \mid \mathrm{B})\end{array}$ & $\begin{array}{c}E V \\
(\mathrm{~A} \mid \mathrm{B})\end{array}$ & $\begin{array}{c}\text { relative } \\
\text { value }\end{array}$ \\
\hline 1 & $\begin{array}{c}\text { baseline probablity } \\
\text { HIGH volume }\end{array}$ & $20 \mid 20$ & $0.2 \mid 0.5$ & $4 \mid 10$ & 0.40 \\
\hline 1 & $\begin{array}{c}\text { baseline probablity } \\
\text { LOW volume }\end{array}$ & $4 \mid 4$ & $0.2 \mid 0.5$ & $0.8 \mid 2$ & 0.40 \\
\hline 1 & $\begin{array}{c}\text { baseline volume } \mathrm{HIGH} \\
\text { probablity }\end{array}$ & $4 \mid 20$ & $0.5 \mid 0.5$ & $2 \mid 10$ & 0.20 \\
\hline 1 & $\begin{array}{c}\text { baseline volume LOW } \\
\text { probablity }\end{array}$ & $4 \mid 20$ & $0.2 \mid 0.2$ & $0.8 \mid 4$ & 0.20 \\
\hline 1 & congruent & $4 \mid 20$ & $0.2 \mid 0.5$ & $0.8 \mid 10$ & 0.08 \\
\hline 1 & incongruent & $4 \mid 20$ & $0.5 \mid 0.2$ & $2 \mid 4$ & 0.50 \\
\hline 2 & $\begin{array}{l}\text { baseline probablity } \\
\text { HIGH volume }\end{array}$ & $20 \mid 20$ & $0.2 \mid 1$ & $4 \mid 20$ & 0.20 \\
\hline 2 & $\begin{array}{c}\text { baseline probablity } \\
\text { LOW volume }\end{array}$ & $4 \mid 4$ & $0.2 \mid 1$ & $0.8 \mid 4$ & 0.20 \\
\hline 2 & $\begin{array}{c}\text { baseline volume HIGH } \\
\text { probablity }\end{array}$ & $4 \mid 20$ & $1 \mid 1$ & $4 \mid 20$ & 0.20 \\
\hline 2 & congruent & $4 \mid 20$ & $0.2 \mid 1$ & $0.8 \mid 20$ & 0.04 \\
\hline 2 & incongruent & $4 \mid 20$ & $1 \mid 0.2$ & $4 \mid 4$ & 1.00 \\
\hline 3 & probability at volume 1 & $4 \mid 4$ & $0.2 \mid 0.5$ & $0.8 \mid 2$ & 0.40 \\
\hline 3 & probability at volume 2 & $10 \mid 10$ & $0.2 \mid 0.5$ & $2 \mid 5$ & 0.40 \\
\hline 3 & probability at volume 3 & $15 \mid 15$ & $0.2 \mid 0.5$ & $3 \mid 7.5$ & 0.40 \\
\hline 3 & probability at volume 4 & $20 \mid 20$ & $0.2 \mid 0.5$ & $4 \mid 10$ & 0.40 \\
\hline 3 & volume at probability 1 & $4 \mid 10$ & $0.2 \mid 0.2$ & $0.8 \mid 2$ & 0.40 \\
\hline 3 & volume at probability 2 & $4 \mid 10$ & $0.5 \mid 0.5$ & $2 \mid 5$ & 0.40 \\
\hline 3 & volume at probability 3 & $4 \mid 10$ & $0.7 \mid 0.7$ & $2.8 \mid 7$ & 0.40 \\
\hline 3 & volume at probability 4 & $4 \mid 10$ & $0.8 \mid 0.8$ & $3.2 \mid 8$ & 0.40 \\
\hline
\end{tabular}

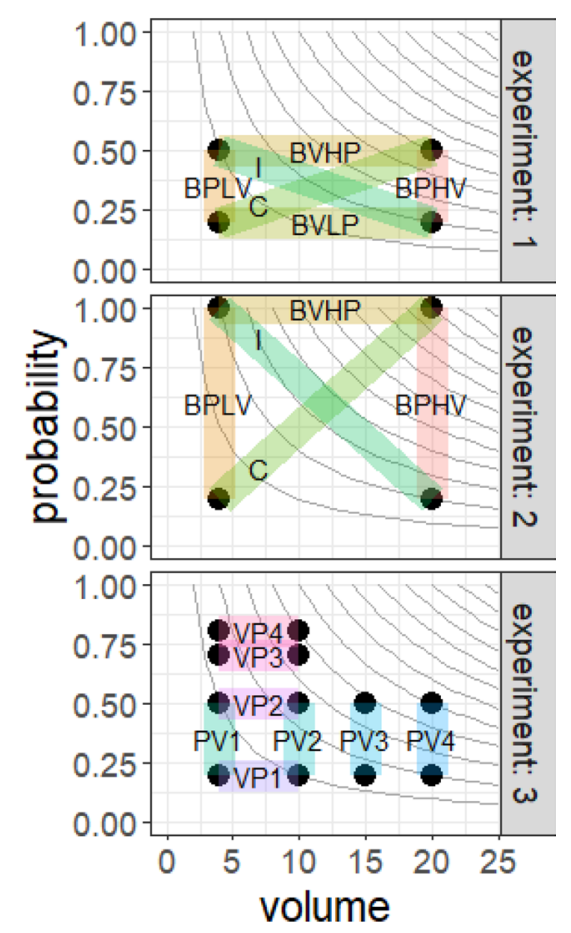

Fig. 2 Overview of the experimental conditions in all four experiments. Options ( $A$ and $B$ ) differed on one or both reward dimensions (reward volume and probability), resulting in different expected values (EV). The black dots give the volume and probability for each option. The transparent segments connect the two options available in each condition. Gray curves give points of equal expected value $\left(\mathrm{EV}=\right.$ volume $\times$ probability). The relative value is $E V_{A} / E V_{B}$. The

following the constraints described above. The reversal condition is potentially harder to learn and may represent the lower bound of choice performance, but its exclusion from the results did not lead to any qualitative changes. Over the 50 total days in the main experiment (twice the number of conditions shown in Fig. 2, because of reversals, plus experiment 4), each mouse experienced each dispenser as a high-profitability dispenser between 11 and 14 times. In the event of an electrical or mechanical malfunction, data from the failed condition and its reversal were discarded and the failed condition was repeated at the end of the experiment, lengthening slightly the duration of the experiment. Such a failure occurred once in cohort 1 , four times in cohort 2 , and did not occur in cohort 3 . After experiments 1 and 2, mice were given another training phase (rewards with $10 \mu \mathrm{L}$ and 0.3 probability) for a single day, before they proceeded with the next experiment. After experiment 3, mice were given water from a standard water bottle for 4 days (with water dispensers inactive), followed by 1 day in the training phase, before proceeding with experiment 4 . At the end of experiment 4 mice were returned to the animal facility. conditions in experiment 4 were identical to those in experiment 1. The baseline for volume at low-probability condition (BVLP) in experiment 1 was not repeated in experiment 2, but, instead, the results from experiment 1 were reused in further analyses. Condition sequences were randomized for each mouse. Volumes shown (in $\mu \mathrm{L}$ ) are for cohorts 1 and 3 . In cohort 2, the volumes were 4.7 instead of 4, 9.4 instead of 10, 14.0 instead of 15 , and 20.3 instead of $20 \mu \mathrm{L}$

\section{Experiment 1}

In the baseline conditions, rewards only differed on one reward dimension (the relevant dimension), but not on the other dimension (the background dimension). For example, in the baseline for probability at low volume (BPLV) condition, both options had the same volume of $4 \mu \mathrm{L}$, but one option had a probability of 0.2 and the other, a probability of 0.5 (Figs. 1b, 2). In the baseline for volume conditions (Fig. 1b), both rewarding options had the same probabilities (either 0.2, baseline for volume at low probability, BVLP; or 0.5 , baseline for volume at high probability, BVHP), but one had a volume of $4 \mu \mathrm{L}$, and the other had a volume of 20 $\mu \mathrm{L}$. Based on previous experiments (Rivalan et al. 2017), we expected a baseline difference between $4 \mu \mathrm{L}$ and $20 \mu \mathrm{L}$ volumes to result in a similar discrimination performance (relative preference for the superior option) compared to a baseline difference between probabilities 0.2 and 0.5 . In the congruent $(\mathrm{C})$ condition, one option was superior to the other on both dimensions (Fig. 1b). Finally, in the incongruent (I) condition, each of the options was superior to the other on one of the reward dimensions, so that the option 
that had the higher volume had the lower probability and vice versa (Fig. 1b). The main goals of this experiment were to (1) test whether the baseline performance when only one dimension was relevant was a good predictor for the discrimination performance in the congruent and incongruent conditions when both dimensions were relevant and (2) whether the trade-off between dimensions affected preference in the incongruent condition. Since the differences on both dimensions were chosen to be of comparable salience (Rivalan et al. 2017), we expected the mean discrimination performance in the incongruent condition to be at chance level (0.5), despite the difference in expected value (Fig. 2).

\section{Experiment 2}

In previous experiments (Rivalan et al. 2017), we had shown that the relative stimulus intensity (i), i.e., the absolute difference between two options divided by their mean (difference/mean ratio), was a good predictor of discrimination performance for both volume and probability differences. Another finding from these experiments was that, at least initially, mice responded less strongly to differences in volume than to differences in probability, despite equivalence in expected values (Rivalan et al. 2017). We aimed to correct for this effect in experiment 1 by selecting options with a higher relative intensity for volume $(4 \mu \mathrm{L}$ vs. $20 \mu \mathrm{L}, i=$ $1.33)$ than for probability ( 0.2 vs. $0.5, i=0.857)$. In experiment 2 , we wanted to test whether mice would exhibit a decreased sensitivity for volume when both reward dimensions had the same relative intensity $(i=1.33)$. Thus, for the conditions in experiment 2 , we simply replaced the 0.5 probability from the conditions in experiment 1 with a probability of 1 (Fig. 2). We did not repeat the BVLP condition, in which both probabilities were set at 0.2 . With the two choice options having the same expected values, we hypothesized that the discrimination performance in the incongruent condition would be at chance level if both dimensions were equally weighed and equally perceived. On the other hand, if mice were less sensitive for volume than for probability differences as in our previous experiments, then the discrimination performance in the incongruent condition should be skewed towards probability $(<0.5)$.

\section{Experiment 3}

In the previous experiments, we used two different baseline conditions for each dimension (BPLV, BPHV, BVLP, and BVHP, Fig. 2), to exhaust all combinations of reward stimuli and balance the experimental design. However, we also wanted to test whether the level of the background dimension despite being the same across choice options nevertheless affected the discrimination performance on the relevant dimension. If mice use a non-compensatory decision rule, we can predict that, regardless of the level of the background dimension, the discrimination performance on the relevant dimension should remain constant. Alternatively, with absolute reward evaluation, the subjective difference between the options is said to decrease as the background (irrelevant) dimension increases and, therefore, the discrimination performance is also expected to decrease (Shafir and Yehonatan 2014). This prediction is derived from the concave shape of the utility function, which is generally assumed to increase at a decreasing rate with the increase in any reward dimension (Kahneman and Tversky 1979; Kenrick et al. 2009; but see also Kacelnik and Brito e Abreu 1998). The same prediction can be made if we assume that motivation decreases with satiety, i.e., the strength of preference decreases under rich environmental conditions (Schuck-Paim et al. 2004), for example at high-reward volume or probability. To test whether the two reward dimensions (volume and probability) interact with each other even when one of them is irrelevant (as background dimension that is the same across choice options), we performed experiment 3 .

The conditions in experiment 3 were chosen to be similar to the baseline conditions in the previous experiments, by having one background and one relevant dimension (Fig. 2). The relevant dimension always differed between the two options. For the probability dimension, we selected the same values of 0.2 and $0.5(i=0.86)$, as in the previous experiments. For the volume dimension, we selected the values of $4 \mu \mathrm{L}$ and $10 \mu \mathrm{L}$ ( $4.8 \mu \mathrm{L}$ and $9.6 \mu \mathrm{L}$ in cohort 2, Fig. 2), because these values have the same relative intensity as the two probabilities. Furthermore, the combination of a higher volume with a probability of 0.8 was expected to result in an insufficient number of visits for analysis. Cohort 2 had different reward volumes due to differences in the pumping process between the two cages used (cage and dispenser system), which also resulted in a lower relative intensity for volume ( $i=0.67$ instead of 0.86 ; we will return to this point in the discussion). There were four different levels for each background dimension (volume and probability, Fig. 2). Each mouse had its own pseudo-random sequence of the eight possible conditions.

\section{Experiment 4}

For laboratory mice that have unrestricted access to a water bottle, the volume of a water reward is not usually a stimulus that predicts reward profitability. In previous experiments (Rivalan et al. 2017), mice had shown an improved discrimination performance for volume over time. This suggests that with experience, mice become more attuned to the relevant reward dimension. To test whether the discrimination performance for one or both dimensions improved over time, we performed experiment 4 , which had the same conditions as experiment 1 (Fig. 2), but with a new pseudo-random 
order. The same mice participated in all experiments (1-4), with about 7 weeks between experiment 1 and experiment 4 .

\section{Data analysis}

Data analysis and simulations were done using $\mathrm{R}$ ( $\mathrm{R}$ Core Team 2020). All data and code are available in the Zenodo repository: https://doi.org/10.5281/zenodo.4223729.

On average (mean $\pm S D$ ), mice made $477 \pm 163$ nose pokes per drinking session (Fig. S1), with a mean proportion of $0.79 \pm 0.1$ nose pokes at the rewarding dispensers. To analyze choices only after mice had some experience with each option (Rivalan et al. 2017), we excluded the first 150 nose pokes at the rewarding dispensers (Figs. S2-S5). We also tried the following alternative approaches: taking the 100 nose pokes between the 151 st and the 251st, taking the last 100 or the last 20 nose pokes, or only taking the nose pokes after the discrimination performance (see below) in two consecutive blocks of 20 nose pokes exceeded the individual mean performance for that drinking session. None of the major results were qualitatively changed with the alternative cut-off points (except for experiment 3 , see discussion), so in the main results, we only report the results with the exclusion of the first 150 nose pokes at the rewarding dispensers.

From the remaining nose poke data, we calculated the discrimination performance for each mouse and each condition of each experiment. Since each condition was repeated twice (initial acquisition and reversal), we calculated the discrimination performance as the total number of nose pokes at the high-profitability dispenser divided by the sum of the total number of nose pokes at the high- and at the low-profitability dispensers. Nose pokes at the non-rewarding dispensers were ignored. In the incongruent condition of experiment 2 in which the profitability was equal (relative value $=1$, Fig. 2), the dispenser with the higher reward volume was treated as the "high-profitability" dispenser. It is important to emphasize that the discrimination performance does not necessarily reflect the capability of mice to distinguish between options, but also depends on other factors such as (over-)training, motivation, and exploratory behavior. Thus, the primary measure in our experiments was the discrimination performance that could develop in one drinking session, controlled for positional biases.

\section{Equivalence tests in experiments 1, 2, and 4}

To investigate how the two reward dimensions contributed towards choice in experiments 1,2 , and 4 , we looked at the contrasts between the baselines (when only one dimension was relevant) to the conditions when the two dimensions were congruent or incongruent to each other. We statistically evaluated these contrasts with the two one-sided procedure (TOST) for equivalence testing (Lauzon and Caffo 2009; Lakens 2017b).

First, we picked a priori a smallest effect size of interest (sesoi) as the difference in discrimination performance of 0.1 units in either direction. This value was chosen based on standard deviations (sd) in discrimination performance observed in previous studies (e.g., Fig. 4 in Rivalan et al. 2017), which ranged from 0.05 to 0.1 . Although discrimination performance is bound by 0 and 1 , most empirical values, especially the differences between two values, are far enough from these bounds so that their distribution approaches the normal. The expected sd of the difference between two normal distributions with sd of 0.1 (we conservatively picked the largest value) is $\sqrt{0.1^{2}+0.1^{2}}=0.141$. With this standard deviation and a sample size of 24 , the equivalence bounds needed to detect equivalence of paired samples with a power of 0.95 are $[-0.1,0.1]$ (powerTOSTpaired.raw function in package TOSTER, Lakens 2017a). The sesoi can be graphically represented as the $[-0.1,0.1]$ interval around the difference of zero, or as $[0.4,0.6]$ around the chance performance of 0.5 .

We then estimated the mean differences and their confidence intervals (CIs) from 1000 non-parametric bootstraps using the smean. $\mathrm{cl}$.boot function in package Hmisc (Harrell and Dupont 2019). For a single equivalence test, the $90 \% \mathrm{CI}$ is usually constructed, i.e., $1-2 \alpha$ with $\alpha=0.05$, because both the upper and the lower confidence bounds are tested against the sesoi (Lauzon and Caffo 2009; Lakens 2017b). This $90 \%$ CI can be fully bounded by the sesoi interval, in which case the observed effect is statistically smaller than any effect deemed worthwhile. In the opposite case, there is no statistical support for equivalence. With conventional null hypothesis testing, the $95 \% \mathrm{CI}$ either does not include the null hypothesis (usually zero), in which case there is a statistically significant difference, or, if it does include the null, the difference is not statistically significant. When combining the equivalence and null hypothesis tests (which can also be done with examination of the $95 \%$ and $90 \%$ confidence intervals), there are four possible outcomes (Lakens 2017b):

1. If the $90 \% \mathrm{CI}$ is fully bounded by the sesoi and the $95 \%$ $\mathrm{CI}$ includes the null, there is statistical support for equivalence.

2. If the $90 \% \mathrm{CI}$ is fully bounded by the sesoi, but the $95 \%$ CI does not include the null, there is statistical support for difference with an effect size smaller than the sesoi. This result can be interpreted as practical equivalence or trivial difference.

3. If the $90 \% \mathrm{CI}$ is not fully bounded by the sesoi, but the 95\% CI includes the null, the result is deemed inconclusive. 
4. If the $90 \% \mathrm{CI}$ is not fully bounded by the sesoi and the 95\% CI does not include the null, there is statistical support for difference.

Therefore, we only considered absolute differences in discrimination performance of at least 0.1 to be of practical significance in our study. Smaller differences, regardless of their statistical significance using other tests, were considered to be trivial.

\section{Linear regression and equivalence tests in experiment 3}

To test whether the background dimension affected discrimination performance, we fitted linear regression models for each mouse and each dimension, with discrimination performance as the dependent variable and background level as the independent variable. The background level was the proportion of the actual value to the maximum of the four values tested, e.g., the background levels for volumes $4,10,15$, and 20 were $0.2,0.5,0.75$, and 1 , respectively. We defined a priori smallest effect size of interest (sesoi), as 0.125 , which is the slope that would result from a difference of 0.1 (the sesoi in experiments 1,2, and 4) in discrimination performance between the smallest and the largest background levels (PV1 and PV4, 0.2 and 1, respectively). A slope estimate (whether positive or negative) within the sesoi interval would allow us to reject an effect of background dimension of 0.125 or larger, which can be interpreted as practically equivalent to an absence of a meaningful effect.

\section{Control of type l error rate}

Researchers have shown that to correct for multiple comparisons in equivalence tests, it suffices to apply a familywise correction of the $\alpha$ for the problematic cases where the type I error is most likely (Davidson and Cribbie 2019), i.e., when equivalence is supported, but the mean difference is close to the sesoi bound. The families of tests, for which multiple comparisons occur in our study, are the eight contrasts in each of experiments 1,2 , and 4 (three families), the tests on the two slopes in experiment 3 , and the six before-after contrasts between experiment 1 and 4 . For each of these five families, the $\alpha$ was divided by $k^{2} / 4$, where $k$ was the number of problematic cases in each family (Caffo et al. 2013). However, the number of problematic cases did not exceed two in any of the test families, which resulted in the corrected $\alpha$ equal to the original value of 0.05 . Furthermore, even with $k$ equal to eight, two, and six (the total number of tests in each test family), only a single result changed from non-equivalent to inconclusive. We therefore report the uncorrected $90 \%$ and $95 \%$ CIs.

\section{Simulations}

To examine whether the behavior of the mice was more consistent with integrative or with non-compensatory rules, we implemented simulations with six different decision rules. We based our decision models on the scalar utility theory (SUT: Kacelnik and Brito e Abreu 1998; Rosenström et al. 2016), which models memory traces for reward amounts (or volumes) as normal distributions rather than point estimates. The scalar property is implemented by setting the standard deviations of these distributions to be proportional to their means. Choice between two options with different volumes can be simulated by taking a single sample from each memory trace distribution and selecting the option with the larger sample.

As previously explained, the discrimination performance for reward probabilities can be reasonably predicted by the relative intensity of the two options (Rivalan et al. 2017). This suggests that the memory traces of reward probabilities also exhibit the scalar property, so that discrimination of small probabilities (e.g., 0.2 vs. $0.5, i=0.86$ ) is easier than discrimination of large probabilities (e.g., 0.5 vs. $0.8, i=$ 0.46 ). Consequently, discrimination (of either volumes or probabilities) when options vary along a single dimension can be predicted by SUT.

\section{Virtual mice}

To extend the basic SUT model for multidimensional choice situations, we implemented six variations that differed in the use of information from the volume and probability dimensions (Table 1), including integrative and non-compensatory models. The information from the different reward dimensions was used to obtain for each choice option a remembered value (utility), which exhibited the scalar property. Choice was simulated by single sampling from the remembered value distributions with means equal to the remembered values and standard deviations proportional to the remembered values.

In an earlier version of the foraging model, mice started without knowledge of the reward properties and learned through Bayesian updating (Foley and Paul 2017). To focus on post-acquisition performance, we removed the first 150 visits, like we did with the empirical data. Analyzing the remembered values of the virtual mice revealed that they had converged on the actual reward values with a small fluctuation around those. For simplicity, here, we decided to simulate only post-acquisition discrimination performance. The virtual mice thus began each experimental condition in a learned state with remembered values equal to the reward dimensions for both choice options and (further) learning was not simulated. Modelling the learning process is outside the scope of this study. 
Table 1 Decision-making models

\begin{tabular}{|c|c|c|c|c|}
\hline Abbreviation & Model & Remembered value & Criterion & $\gamma$ \\
\hline sev & Scalar expected value & $\pi \mathcal{N}(v, \gamma v)$ & - & 1.05 \\
\hline 2 scal & Two-scalar & $\mathcal{N}(\pi, \gamma \pi) \times \mathcal{N}(\nu, \gamma v)$ & - & 0.65 \\
\hline rnonc & Randomly non-compensatory & $\mathcal{N}(r, \gamma r)$ & $\theta_{v}=0.5$ & 0.05 \\
\hline wta & Winner-takes-all & $\mathcal{N}(r, \gamma r)$ & $\theta=1$ & 0.7 \\
\hline pfirst & Probability first & $\mathcal{N}(r, \gamma r)$ & $\begin{array}{l}\text { If } s(\pi)>0.8 \text { then } r=\pi \text {, } \\
\text { if } s(v)>0.8 \text { then } r=v \text {, } \\
\text { otherwise } \theta=0.5\end{array}$ & 0.95 \\
\hline vfirst & Volume first & $\mathcal{N}(r, \gamma r)$ & $\begin{array}{l}\text { If } s(v)>0.8 \text { then } r=v \text {, } \\
\text { if } s(\pi)>0.8 \text { then } r=\pi \\
\text {, otherwise } \theta=0.5\end{array}$ & 0.5 \\
\hline
\end{tabular}

$\pi$-probability estimate; $v$-volume estimate; $\gamma$-coefficient of variation; $r$-either $v$ or $\pi$ depending on the criterion; $\theta_{v}$-probability of selecting the volume dimension; $\theta$-probability of selecting the dimension with the higher salience; $s(r)$-salience of dimension $r$, calculated as $\frac{\max (r)-\min (r)}{\bar{r}}$, where $\bar{r}$ is the arithmetic mean of $r$ over all options
From its memory traces, a virtual mouse generated one remembered value distribution for each choice option, according to one of six different rules (see Table 1). Action selection was then implemented by taking a single sample from each distribution and selecting the option with the larger sample.

\section{Decision-making models}

1. Scalar expected value model. There is a single memory trace for each option and it consists in the simple product of the estimate for the volume and the estimate for the probability (expected value). The scalar property is implemented as $\pi \mathcal{N}(v, \gamma v)$, where $\pi$ is the probability estimate. $\mathcal{N}(\mu, \sigma)$ is a normal distribution with mean $\mu$ and standard deviation $\sigma, v$ is the volume estimate, and $\gamma$ is a free parameter, the coefficient of variation. This model thus utilizes information from all dimensions for every decision.

2. Two-scalar model. There are traces for each dimension for every option, where each trace exhibits the scalar property independently and the value is obtained by simple multiplication of the traces for each dimension: $\mathcal{N}(\pi, \gamma \pi) \times \mathcal{N}(v, \gamma v)$. This model also utilizes information from all dimensions for every decision. Although it allows each dimension to have its own scalar factor, e.g., $\gamma_{\pi} \neq \gamma_{v}$, for simplicity, we assume that they are both equal.

The memory traces in the remaining models are identical to the traces in the two-scalar model, but these models usually consider only a single dimension.

\section{Randomly non-compensatory model. Each decision is}

4. Winner-takes-all model. Each decision is based only on the dimension with the highest salience. The salience for a vector of estimates from memory traces (mean values) along one dimension, e.g., volume $v=\left(v_{1}, v_{2}, \ldots, v_{n}\right)$, is calculated as $\frac{\max (v)-\min (v)}{\bar{v}}$, where $n$ is the number of options. In the case of $n=2$, the salience is equivalent to the previously described relative intensity measure. For dimensions of equal salience, the model reverts to random choice.

The last two models are examples of a lexicographic rule, in which the dimensions are checked in a specific order. If the salience of a dimension is higher than a given threshold, then a decision is made based only on this dimension. Otherwise, the next-order dimension is checked. If all dimensions have saliences below the threshold, the model reverts to random choice. The value of the threshold was set at 0.8 , the psychometric function threshold for probability (Rivalan et al. 2017), but we also performed sensitivity analyses on the threshold values (Figs. S8, S9).

5. Probability first model. Probability is checked first and then volume.

6. Volume first model. Volume is checked first and then probability.

\section{Environment}

Each of the experimental conditions was recreated in the simulations as a binary choice task between the highprofitability and the low-profitability options. We did not simulate the two non-rewarding options. Upon a visit by a virtual mouse, a choice option would deliver a reward with its corresponding volume and probability (Fig. 2). The virtual environment was not spatially and temporally explicit. Thus, no reversal conditions were simulated and the test of 
each experimental condition consisted in a sequence of 100 choices. All experimental conditions in all four experiments were tested.

\section{Model fits}

All models described above share the same free parameter, the scalar factor $\gamma$. To obtain baseline estimates for $\gamma$ for each of the models (Table 1), we focused on the probability baseline discrimination performances of all mice in experiments 1 and 4 (baseline conditions BPLV and BPHV). We performed a grid search sensitivity analysis by varying $\gamma$ with steps of 0.05 in the range of $(0.05,2)$. We generated 100 decisions by 100 mice for each cell in this grid and then used locally weighted scatterplot smoothing (loess) to fit a model for each condition. The free parameter values that resulted in the smallest RMSEs compared to the observed baseline data were selected for the comparison of the six models (Table 1). We also performed a sensitivity analysis for different values of the free parameters $\theta_{v}$ in the randomly non-compensatory model and of the thresholds for volume and probability in the volume first and probability first models, in the range of $(0,1)$, with a step of 0.05 . The resulting free parameter estimates (across animals) were then used in out-of-sample tests of the six models. For each of the experimental conditions in the four experiments (Fig. 2) and for each of the six models, we simulated 100 choices by 100 (identically parametrized) mice. Over the 100 choices, we calculated the discrimination performance for each mouse and then used the median of the individual discrimination performances as the model prediction. We then quantified the model fits to the empirical data by calculating root-mean-square-errors (RMSE), excluding the BPLV and BPHV conditions in experiments 1 and 4. Finally, we ranked the models by their RMSE scores.

\section{Results}

\section{Experiment 1: mice consistently preferred the more profitable option, even with a trade-off between reward probability and reward volume}

Generally, compared to the baselines, mice showed an increase in discrimination performance in the congruent condition and a decrease in performance in the incongruent condition (Fig. 3a, b). The only exception was the C-BVHP contrast, which had an effect size smaller than the sesoi $(0.05,95 \%$ CI $=[0.02,0.09])$. Furthermore, when we excluded cohort 2 , the C-BVHP contrast became equivalent to zero $(0.01,95 \% \mathrm{CI}=$ $[-0.02,0.04])$. Contrary to our expectations based on previous work, the trade-off between volume and probability chosen for this experiment did not abolish preference for the higher volume option in the incongruent condition, with a discrimination performance significantly higher than the chance level of 0.5 $(0.57,95 \% \mathrm{CI}=[0.51,0.64]$, Fig. 3c $)$. However, we again observed very different behavior in cohort 2 , which showed a preference for the higher probability option (Fig. 3c). Thus, at least for mice in cohorts 1 and 3 , in the incongruent condition, there was a preference for the more profitable option and the subjective contrast in probability was not stronger than the subjective contrast in volume.

\section{Experiment 2: some evidence for equal weighing of reward probability and reward volume}

Similar to experiment 1 , in experiment 2 , mice showed an increase in discrimination performance in the congruent condition (with one exception) and a decrease in performance in the incongruent condition (Fig. 4a, b). This time, the exception was the C-BPLV contrast, which was equivalent to $0(0.02,95 \% \mathrm{CI}=[-0.01,0.04])$. Although the discrimination performance in the incongruent condition was again different from $0.5(0.41,95 \% \mathrm{CI}=[0.35,0.47])$, it was lower than chance, thus skewed towards probability (Fig. 4b). However, when the data from cohort 2 were excluded, the discrimination performance became equivalent to $0.5(0.48,95 \% \mathrm{CI}=[0.42,0.54])$. We return to the differences between cohorts in the discussion. Thus, it appears that, at least for mice in cohorts 1 and 3, the subjective contrasts in volume and probability were equal and no reward dimension seemed to have priority over the other.

\section{Experiment 3: probability discrimination decreased with an increase in reward volume, but volume discrimination was not affected by changes in reward probability}

The results of experiment 3 show that the discrimination performance for probability decreased with increasing volumes, although the effect size was small $(-0.1,95 \% \mathrm{CI}$ $=[-0.16,-0.06]$, without cohort $2:(-0.147,95 \% \mathrm{CI}=$ [-0.212, -0.088$]$, Fig. 5). In contrast, the discrimination performance for volume was practically independent from probability as the background dimension, since the estimate for the slope was smaller than the sesoi $(0.06,95 \% \mathrm{CI}=$ [-0.01, 0.14], Fig. 5). Without cohort 2, the slope estimate for the volume dimension was still small, but significantly positive $(0.072,95 \% \mathrm{CI}=[0.009,0.139])$. These results partially support the hypothesis that decision-makers may ignore a reward dimension along which options do not vary.

\section{Experiment 4: mice improved their volume discrimination over time}

In the comparison between experiment 1 and experiment 4 , mice showed an improved discrimination performance 

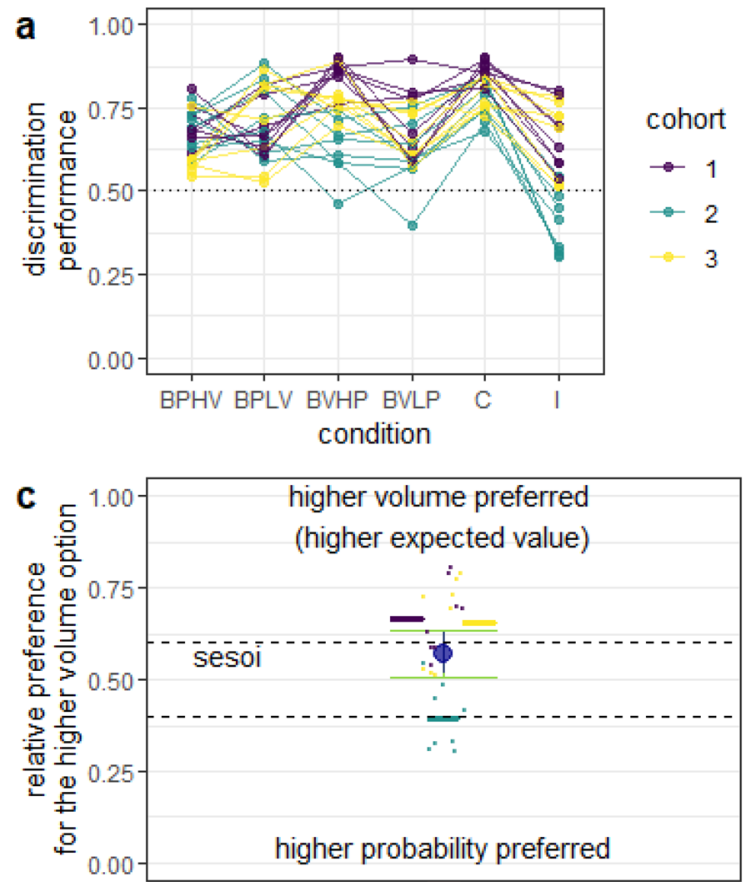

Fig. 3 Discrimination performance in experiment 1. a Each dot is the mean discrimination performance of an individual mouse over two presentations of the same condition (initial acquisition and reversal). Experimental conditions are described in detail in Fig. 2. The discrimination performance gives the relative visitation rate of the more profitable option, or, in the incongruent condition, the option with the higher volume. Dotted line gives the chance level (0.5). Data are shown in different colors for three different cohorts of eight mice each (total $n=24$ ). Data from the same individuals are connected with lines. Cohort 2 (green) was tested in a different cage set-up than the other two (see Methods for details). b Difference between discrimination performance in the baseline conditions and in the congruent and incongruent conditions. Dots show the individual differences in discrimination performance for the given conditions of each individual mouse (color-coded for cohort as in a). Positive differences indicate an increase in performance and negative differ-

in both volume baselines, as well as in the incongruent and BPLV conditions (Fig. 6). There was only a trivial improvement in the congruent condition (Fig. 6). When we applied a familywise error control procedure, only the BPLV result changed from an increase to inconclusive and the congruent condition, from trivial to equivalent. Thus, consistent with our prior findings, mice improved their volume discrimination over time.

The discrimination performance in the congruent condition was better than either of the probability baselines, but equivalent to the volume baselines (Fig. 7a, b). For cohorts 1 and 3, the discrimination performance in the incongruent condition was lower than in any of the four baselines, but the difference from the volume baselines was smaller (Fig. 7b). Cohort 2 showed the opposite pattern (Fig. 7b). Finally, compared to experiment 1 , the influence of the volume dimension on choice and the discrepancy between

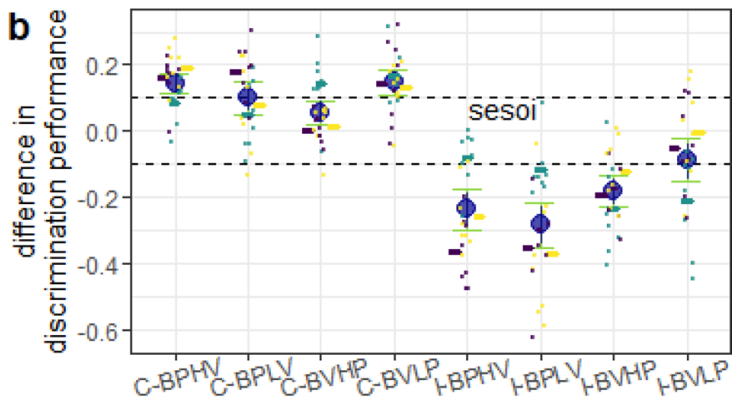

ences-a decrease in performance, compared to the baseline. Horizontal colored lines give the cohort means. Large blue circles give the means and the blue vertical lines the $90 \%$ confidence intervals from non-parametric bootstraps. The smallest effect size of interest (sesoi) is represented by the dashed lines. Green whiskers give the $95 \% \mathrm{CI}$ from non-parametric bootstraps. When the blue confidence intervals lie completely within the sesoi interval, there is statistical support for equivalence (Lakens 2017b). The discrimination performance in the incongruent condition was calculated as the relative preference for the higher probability dispenser when contrasted with the probability baselines (e.g., I-BPLV) and for the higher volume dispenser when contrasted with the volume baselines (e.g., I-BVHP). c Discrimination performance in the incongruent condition. Dashed lines give the sesoi around chance level performance. Remaining notation is the same as in b. In this experiment, the option with the higher volume was also the more profitable option

cohort 2 and the other cohorts were even more pronounced (Fig. 7c).

\section{Decision models of two-dimensional choice suggest that mice initially relied on both reward volume and reward probability, but then developed a bias for reward volume}

There was no single model that could best explain the choice of the mice in all four experiments, but the scalar expected value, two-scalar, and winner-takes-all models were in the top-three performing models most frequently (Tables 1, 2, Figs. S10-S13). However, due to the unexpected differences in performance between cohort 2 and the other cohorts (e.g., Fig. S13), we also ranked the models separately for the different mouse groups, depending on which cage they 

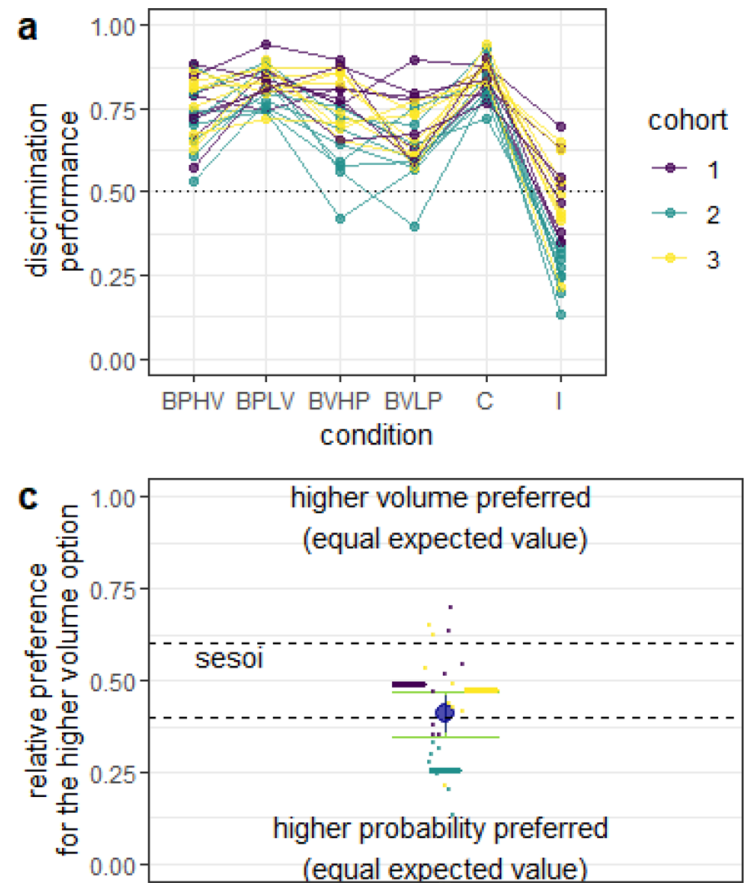

Fig. 4 Discrimination performance in experiment 2. Same notation as in Fig. 3. a Discrimination performance in all conditions. b Difference between discrimination performance in the baseline conditions and in the congruent and incongruent conditions. The discrimination performance in the incongruent condition was calculated as the relative preference for the higher probability dispenser when

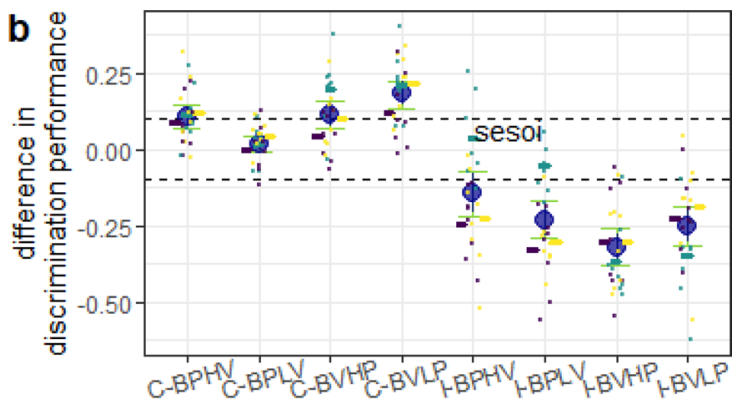

contrasted with the probability baselines (e.g., I-BPLV) and for the higher volume dispenser when contrasted with the volume baselines (e.g., I-BVHP). c Discrimination performance in the incongruent condition. In this experiment, both options were equally profitable and had the same expected value

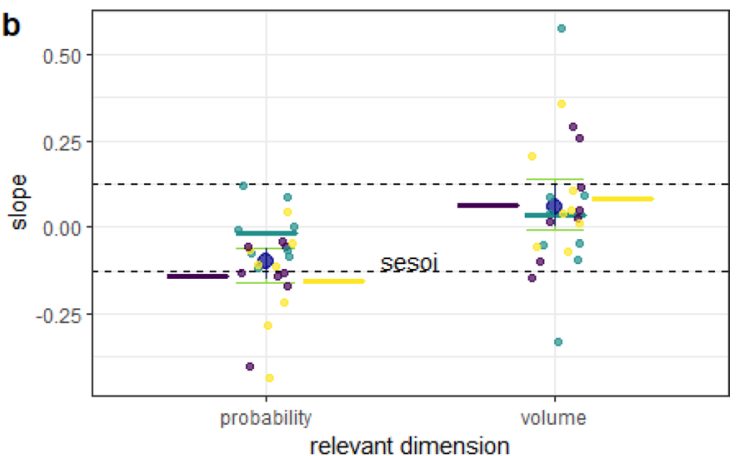

ear fits. Cohort 2 (green) was tested in a different cage set-up than cohorts 1 and 3 (see Methods for details). b Each colored dot represents the individual slope of one line in a. The smallest effect size of interest (sesoi, dashed lines) was determined to be the slope $(0.125)$ that would have resulted in a difference in discrimination performance of 0.1 , from the lowest to the highest level of the background dimension (from PV1 to PV4 in (a)). Large blue circles give the means and the blue vertical lines the 90\%-confidence intervals from non-parametric bootstraps. Green whiskers give the $95 \%$ CI from non-parametric bootstraps. Horizontal colored lines give the cohort means 


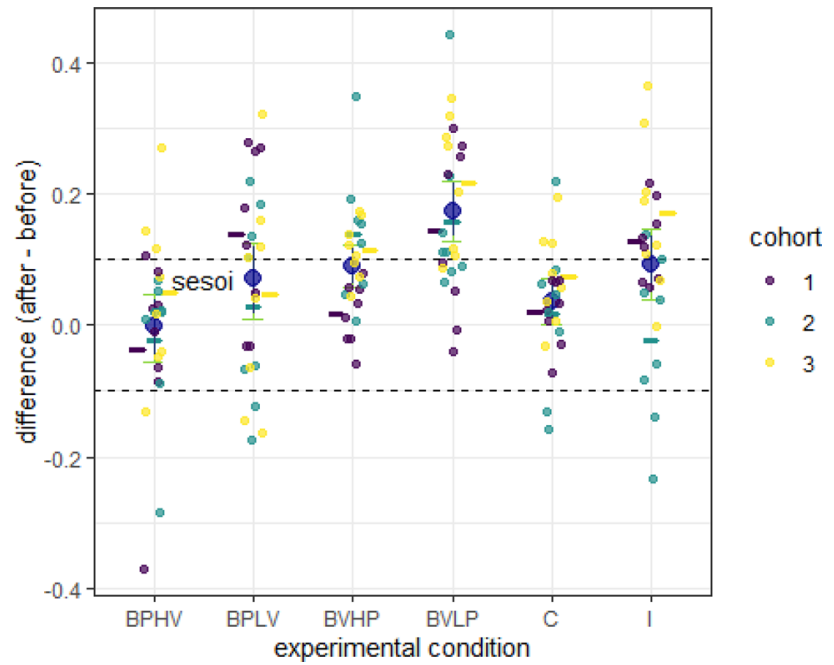

Fig. 6 Difference in discrimination performance between identical conditions in experiment 1 and experiment 4 . Same notation as in in Fig. 3. The sequence of conditions was pseudo-random in each experiment and different for each individual. Positive differences indicate an increase in discrimination performance with time. Mice were 7 weeks old at the beginning of experiment 1 and 13-14 weeks old at the beginning of experiment 4 . The discrimination performance in the incongruent condition was calculated as the relative preference for the higher volume dispenser

performed the experiments in (cohorts 1 and 3 in cage 1 and cohort 2 in cage 2 ). Indeed, two different patterns emerged for the different cages. For the two cohorts in cage 1, scalar expected value and two scalars were the best supported models, followed by the winner-takes-all and volume first models (Table 3). Notably, the volume first model was the bestperforming model in the later experiments 3 and 4 , but the worst model in the earlier experiments 1 and 2 . In contrast, the probability first model was the best supported model for cohort 2 , followed by the randomly non-compensatory, winner-takes-all, and scalar expected value models (Table 4).

\section{Discussion}

The foraging choices of the mice in this study provide evidence both for and against full integration of reward volume and probability. In the first two experiments, most mice differed in discrimination performance (increased or decreased) in the conditions in which both reward dimensions were simultaneously relevant (congruent and incongruent conditions) compared to the baselines, in which only one of the two dimensions was relevant at a time (Figs. 3, 4). Consequently, the best supported models for these two experiments (cohort 2 excluded, see discussion about differences between cohorts below) were the models that made use of the full information from both reward dimensions (sev, 2 scal), or from the dimension that was subjectively more salient (wta, Table. 3). Although these models were good predictors of choices in experiments 3 and 4, as well, the best-performing model in experiments 3 and 4 was the one that considered the volume dimension first and the probability dimension only if differences on the volume dimension were insufficient to reach a decision (Table 3 ). Thus, it appears that mice initially used information from all reward dimensions without bias and with experience started to rely more on one reward dimension and disregarded the other when both dimensions differed between choice options. Interestingly, in human development, the use of integrative decision rules has also been shown to decrease with age (Jansen et al. 2012).

In similar and more complex choice situations when options vary on several dimensions, an animal has no immediate method of distinguishing the relevant from the background dimensions. Instead, it must rely on its experience over many visits before it can obtain information about the long-term profitability associated with the different reward dimensions. Under such circumstances, a decision rule that considers all or the most salient reward dimensions initially and prioritizes dimensions based on gathered experience can be profitable without being too computationally demanding. Indeed, with the particular experimental design in this study, a mouse using a "volume first" priority heuristic would have preferentially visited the more profitable option (whenever there was one) in every single experimental condition, including the incongruent conditions.

\section{Scalar property considerations}

An alternative explanation of our main results is that the mice used the "volume first" heuristic from the beginning of the experiment, but only became better at discriminating volumes (their coefficient of variation $\gamma$ decreased) in the last two experiments. This interpretation is supported by the comparison between experiments 1 and 4 (Fig. 6), as well as from previous experiments (Rivalan et al. 2017), in which mice improved their volume discrimination over time. However, it is not possible with these data to distinguish whether the effect was caused by training or maturation. Perhaps an increase in mouth capacity (Vora et al. 2016) or, potentially, in the number of acid-sensing taste receptors (Zocchi et al. 2017) due to growth and maturation could allow adult mice to better discriminate water volumes. We assumed that mice consumed all water without spilling, but perhaps less-experienced mice spill some water. Alternatively, with prolonged training, mice might transition from goal directed strategies to egocentric or habitual responses (Packard and McGaugh 1996; Kosaki et al. 2018; and in mice: Kleinknecht et al. 2012). Comparing the discrimination performance of older untrained and younger trained mice would help clarify this confound. 

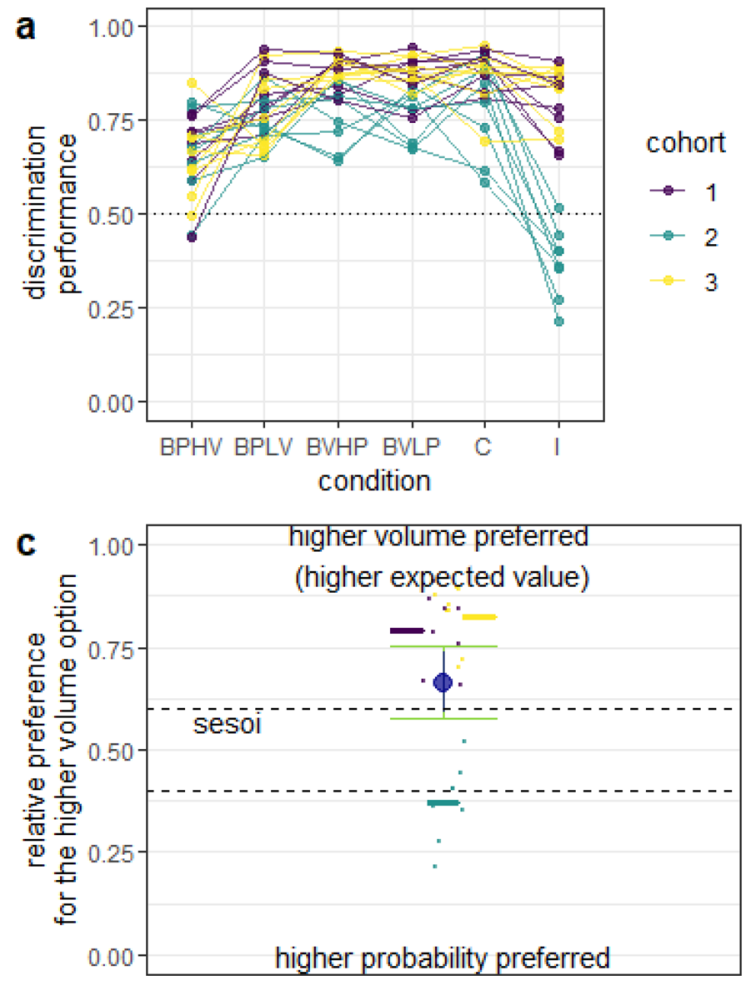

Fig. 7 Discrimination performance in experiment 4, with identical conditions to experiment 1. Same notation as in Fig. 3. a Discrimination performance in all conditions. b Difference between discrimination performance in the baseline conditions and in the congruent and incongruent conditions. The discrimination performance in the incongruent condition was calculated as the relative preference for

Table 2 Best-performing models ranked by root-mean-square errors (RMSE)

\begin{tabular}{lllll}
\hline Experiment & & & & \\
\hline Rank & 1 & 2 & 3 & 4 \\
\hline 1 & sev & sev & vfirst & 2 scal \\
2 & 2 scal & 2 scal & sev & wta \\
3 & wta & wta & 2 scal & sev \\
4 & rnonc & pfirst & wta & vfirst \\
5 & pfirst & rnonc & pfirst & rnonc \\
6 & vfirst & vfirst & rnonc & pfirst \\
\hline
\end{tabular}

The increase in discrimination performance for volume between experiments 1 and 4 (Fig. 6) suggests that the scalar property only approximately holds, and that the $\gamma$ (coefficient of variation) for volume is not truly constant over a long period of time. This can be seen as evidence against the scalar expected value model, which assumes that the same coefficient of variation affects performance along each reward dimension. Instead, the improving volume discrimination supports a version of the two-scalar model, in which there are two different scalars $\left(\gamma_{\pi} \neq \gamma_{v}\right)$. Alternatively, there

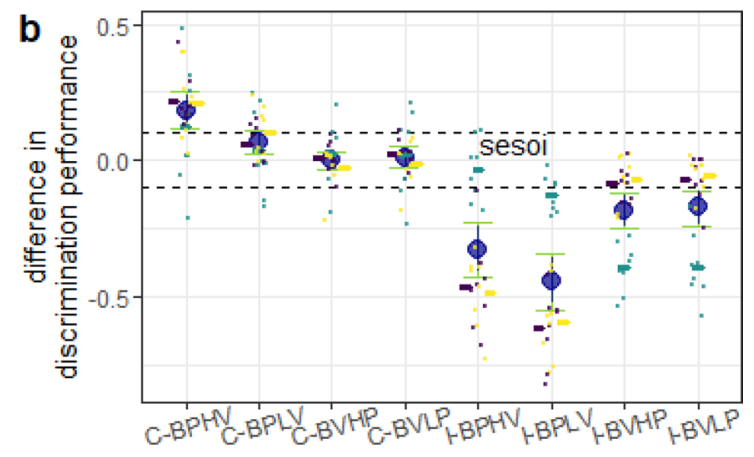

the higher probability dispenser when contrasted with the probability baselines (e.g., I-BPLV) and for the higher volume dispenser when contrasted with the volume baselines (e.g., I-BVHP). c Discrimination performance in the incongruent condition. In experiments 1 and 4 , the option with the higher volume was also the more profitable option. Compare to Fig. 3

Table 3 Best-performing models ranked by root-mean-square errors (RMSE) for cohorts 1 and 3

\begin{tabular}{lllll}
\hline Experiment & & & & \\
\hline Rank & 1 & 2 & 3 & 4 \\
\hline 1 & sev & 2 scal & vfirst & vfirst \\
2 & 2 scal & sev & sev & 2 scal \\
3 & wta & wta & 2 scal & wta \\
4 & rnonc & rnonc & wta & sev \\
5 & pfirst & pfirst & rnonc & rnonc \\
6 & vfirst & vfirst & pfirst & pfirst \\
\hline
\end{tabular}

might be only one scalar, associated with dynamic relative weights of the two dimensions (which can be implemented as a changing $\theta_{v}$ in the randomly non-compensatory model, Fig. S7). Yet, another model extension that can account for the improving volume discrimination would be to introduce an explicit sampling (exploration-exploitation balance) method (Sih and Del 2012; Nachev and Winter 2019). In natural conditions, reward dimensions rarely remain stable over time and foragers can benefit from making sampling choices to gather information about the current state of 
Table 4 Best-performing models ranked by root-mean-square errors (RMSE) for cohort 2

\begin{tabular}{lllll}
\hline Experiment & & & & \\
\hline Rank & 1 & 2 & 3 & 4 \\
\hline 1 & pfirst & pfirst & pfirst & pfirst \\
2 & rnonc & rnonc & wta & rnonc \\
3 & sev & sev & 2 scal & wta \\
4 & wta & 2 scal & sev & 2scal \\
5 & 2 scal & wta & rnonc & sev \\
6 & vfirst & vfirst & vfirst & vfirst \\
\hline
\end{tabular}

the environment. Thus, not all choices need to be based on expected values and individuals may differ in their sampling rates (Sih and Del 2012; Rivalan et al. 2017; Nachev and Winter 2019). With such an implementation, it is not the scalar but the frequency of sampling visits that changes over time, causing differences in discrimination performance. The biggest challenge is that when it comes to volumes and probabilities, no direct method of interrogating an animal's estimate, and coefficient of variation exist, so that researchers have to infer these values from choice behavior, which is also affected by motivation, learning, and sampling frequency. In contrast, when it comes to time intervals, the peak procedure gives us a more direct measurement of the time estimation of animal subjects (Kacelnik and Brito e Abreu 1998).

\section{Interaction between dimensions and non-compensatory decision-making}

Although mice were practically equally good at discriminating volume rewards at each different probability, the discrimination of probabilities decreased at higher volumes (Fig. 5; the estimated effect size was a decrease of 0.12 between a volume background at $4 \mu \mathrm{L}$ and at $20 \mu \mathrm{L}$ ). This suggests that the two dimensions interact with each other. Absolute reward evaluation (Shafir 1994; Shafir and Yehonatan 2014) and state-dependent evaluation (SchuckPaim et al. 2004) are both consistent with this decrease in discrimination performance, but not with the small positive effect in the conditions in which the probability was the background dimension. With comparable expected values (Fig. 2) between the two series of conditions, these hypotheses make the same predictions regardless of which dimension is relevant and which is background. An alternative explanation is that arriving at a good estimate of probability requires a larger number of visits, and when the rewards are richer (of higher volume), mice satiate earlier and make a smaller total number of visits, resulting in poorer estimates of the probabilities and poorer discrimination performance. Consistent with this explanation, mice made on average ( \pm
SD) $474 \pm 199$ nose pokes at the relevant dispensers at $4 \mu \mathrm{L}$, but only $306 \pm 64$ nose pokes at $20 \mu \mathrm{L}$ (Figs. S1, S4: PV1 and PV4, respectively). Furthermore, when we controlled for the number of nose pokes by only analyzing the nose pokes between the 151st and 251st, the effect of volume on probability discrimination became equivalent to zero, suggesting that further learning after the 150st nose poke could have led to an improved discrimination performance. At the same time, controlling for the number of nose pokes also led to a significant (but small) positive effect of probability on volume discrimination (slope estimate $0.9,95 \% \mathrm{CI}=[0.01$, $0.18]$ ). This also suggests that at 0.2 probability, it took mice more than 150 nose pokes to reach the same discrimination performance for volumes observed at probabilities higher than 0.2 (Fig. S4). These were the only qualitative changes caused by taking an alternative cut-off point rather than simply removing the first 150 nose pokes to the rewarding dispensers.

As mentioned earlier, researchers have proposed that with absolute reward evaluation, the difference/mean ratio in an experimental series like our experiment 3 should decrease with the increase of the background dimension, leading to a decrease in the proportional preference for the highprofitability alternative, i.e., discrimination performance (Shafir and Yehonatan 2014). However, this is only the case if the difference is calculated from the relevant dimension and divided by the mean utility. We suggest that both the difference and the mean should be calculated from the same entity, either utility or one of the reward dimensions. When, as in our sev and 2 scal models 1 , we calculate utility by multiplying the estimates for each dimension together, the difference/mean ratio of the utility does not change with the change in the background dimension between treatments. In fact, none of our models in experiment 3 exhibited an effect of the background dimension on the discrimination performance, with all slopes equivalent to zero (Fig. S14). Thus, our results also show that absolute reward evaluation does not necessarily predict an effect of background dimension on discrimination performance.

\section{Difference between cohorts}

Our results revealed some striking differences in behavior between cohort 2 and cohorts 1 and 3 (most obvious in Fig. 7). The most likely explanation for this is an effect of the specific experimental apparatus. As explained in the section "Methods", the precision of the reward volumes was lower in cage 2, which housed cohort 2. However, it is unlikely that such a small magnitude of the difference $\left(0.33 \pm 0.03 \mu \mathrm{L} \mathrm{step}^{-1}\right.$ in cage 1 vs. $1.56 \pm 0.24 \mu \mathrm{L} \mathrm{step}^{-1}$ in cage 2) could influence volume discrimination to the observed extent. Future experiments can address this issue by specifically manipulating the reliability of the volume 
dimension using the higher precision pump. Instead, we suspect that the difference between cohorts might have been caused by the acoustic noise and vibrations produced by the stepping motors of the pumps. The pump in cage 1 was much louder, whereas the one in cage 2 was barely audible (to a human experimenter). This could have made it harder for mice in cage 2 to discern whether a reward was forthcoming, which could have influenced their choices (Ojeda et al. 2018). As a result, mice in cage 2 waited longer before leaving the dispenser during unrewarded nose pokes (Fig. S15). This potentially costly delay might have increased the relative importance of the probability dimension (decreased $\theta_{v}$ ), resulting in the observed discrimination performance in cohort 2. Furthermore, the same line of reasoning can also explain the improving volume discrimination: from the first to the fourth experiment, there was a shift towards shorter unrewarded nose poke durations in the loud cage (cohorts 1 and 3, Fig. S15), suggesting that mice had learned over time to abort the unrewarded visits. This could have decreased the relative importance of the probability dimension (increased $\theta_{v}$ ), resulting in better volume discrimination. In an unrelated experiment, we tested two cohorts of mice in both cages simultaneously and then translocated them to the other cage. The results demonstrated that differences in discrimination performance were primarily influenced by cage and not by cohort (Nachev, in prep.). Thus, the sound cue associated with reward delivery may be an important confounding factor in probability discrimination in mice, as it provides a signal for the reward outcome (Ojeda et al. 2018).

\section{Conclusion}

In summary, our results show that mice could integrate reward volume and reward probability, which allowed them to select the more profitable option when the two reward dimensions varied independently. The resulting partial preference was consistent with SUT. However, we also found that, with time, mice improved their performance in volume (but not as much in probability) discrimination tasks and their choices became more consistent with a non-compensatory decision rule, in which volume is evaluated before probability. Finally, we found that mice could discriminate the same pair of probabilities better when reward volumes were smaller, but changes in the reward probability did not seem to affect their volume discrimination performance.

Supplementary Information The online version supplementary material available at https://doi.org/10.1007/s10071-021-01482-8.

Acknowledgements We thank Miléna Brunet, Alexia Hyde, and Sabine Wintergerst for data acquisition, Katja Frei for assistance with the mice, Alexej Schatz for programming of the control software, and our colleagues of the Winter lab for a fruitful discussion. We also thank Noam Miller, Daniël Lakens, and two anonymous reviewers for their helpful comments and suggestions for improving the manuscript.

Author Contributions VN: conceptualization, methodology, software, formal analysis, data curation, writing — original draft, writing — review and editing, visualization, supervision, project administration. MR: methodology, writing - review and editing, and supervision. YW: resources, methodology, writing-review and editing, and supervision.

Funding Open Access funding enabled and organized by Projekt DEAL.

Availability of data and materials All data and code are available in the Zenodo repository: https://doi.org/10.5281/zenodo.4223729.

\section{Declarations}

Funding No specific funding.

Conflict of interest YW owns PhenoSys equity.

Ethics approval The experiments were conducted under the supervision and with the approval of the animal welfare officer heading the animal welfare committee at Humboldt University. Experiments followed national regulations in accordance with the European Communities Council Directive 10/63/EU.

Code availability All data and code are available in the Zenodo repository: https://doi.org/10.5281/zenodo.4223729.

Open Access This article is licensed under a Creative Commons Attribution 4.0 International License, which permits use, sharing, adaptation, distribution and reproduction in any medium or format, as long as you give appropriate credit to the original author(s) and the source, provide a link to the Creative Commons licence, and indicate if changes were made. The images or other third party material in this article are included in the article's Creative Commons licence, unless indicated otherwise in a credit line to the material. If material is not included in the article's Creative Commons licence and your intended use is not permitted by statutory regulation or exceeds the permitted use, you will need to obtain permission directly from the copyright holder. To view a copy of this licence, visit http://creativecommons.org/licenses/by/4.0/.

\section{References}

Akre KL, Johnsen S (2014) Psychophysics and the evolution of behavior. Trends Ecol Evol 29(5):291-300. https://doi.org/10.1016/j. tree.2014.03.007

Bateson M, Healy SD, Hurly TA (2003) Context-dependent foraging decisions in rufous hummingbirds. Proc R Soc B Biol Sci 270(1521):1271-6. https://doi.org/10.1098/rspb.2003.2365

Brandstätter E, Gigerenzer G, Hertwig R (2006) The priority heuristic: making choices without trade-offs. Psychol Rev 113(2):409-32. https://doi.org/10.1037/0033-295X.113.2.409

Caffo B, Lauzon C, Röhmel J (2013) Correction to 'Easy multiplicity control in equivalence testing using two one-sided tests'. Am Stat 67(2):115-16. https://doi.org/10.1080/00031305.2012.760487

Constantinople CM, Piet AT, Brody CD (2019) An analysis of decision under risk in rats. Curr Biol 29(12):2066-2074.e5. https://doi.org/ 10.1016/j.cub.2019.05.013 
Davidson H, Cribbie RA (2019) A more powerful familywise error control procedure for evaluating mean equivalence. Commun Stat Simul Comput. https://doi.org/10.1080/03610918.2018.1530783

Foley BR, Marjoram P (2017) Sure enough: efficient Bayesian learning and choice. Anim Cogn 20(5):867-80. https://doi.org/10.1007/ s10071-017-1107-5

Harrell FE, Dupont C (2019) R Package Hmisc: Harrell Miscellaneous. https://www.CRAN.R-project.org/package=Hmisc. Accessed 28 Feb 2021

Houston AI, McNamara JM, Steer MD (2007) Violations of transitivity under fitness maximization. Biol Lett 3(4):365-67. https://doi.org/ 10.1098/rsbl.2007.0111

Hunt LT, Dolan RJ, Behrens TEJ (2014) Hierarchical competitions subserving multi-attribute choice. Nat Neurosci 17(11):1613-22. https://doi.org/10.1038/nn.3836

Jansen BRJ, van Duijvenvoorde ACK, Huizenga HM (2012) Development of decision making: sequential versus integrative rules. J Exp Child Psychol 111(1):87-100. https://doi.org/10.1016/j.jecp. 2011.07.006

Kacelnik A (1984) Central place foraging in starlings (Sturnus vulgaris). I. Patch residence time. J Anim Ecol 53(1):283-99. https:// doi.org/10.2307/4357

Kacelnik A (2006) Meanings of rationality. In: Hurley SL, Nudds M (eds) Rational animals? Oxford University Press, Oxford

Kacelnik A, Brito e AF (1998) Risky choice and Weber's law. J Theor Biol 194(2):289-98. https://doi.org/10.1006/jtbi.1998.0763

Kahneman D, Tversky A (1979) Prospect theory: an analysis of decision under risk. Econometrica 47(2):263-91. https://doi.org/10. 2307/1914185

Katsikopoulos KV, Gigerenzer G (2008) One-reason decision-making: modeling violations of expected utility theory. J Risk Uncertainty 37(1):35-56. https://doi.org/10.1007/s11166-008-9042-0

Kenrick DT, Griskevicius V, Sundie JM, Li NP, Li YJ, Neuberg SL (2009) Deep rationality: the evolutionary economics of decision making. Soc Cogn 27(5):764-85. https://doi.org/10.1521/soco. 2009.27.5.764

Kleinknecht KR, Bedenk BT, Kaltwasser SF, Gruenecker B, Yen Y-C, Czisch M, Wotjak CT (2012) Hippocampus-dependent place learning enables spatial flexibility in c57bl6/n mice. Front Behav Neurosci. https://doi.org/10.3389/fnbeh.2012.00087

Kosaki Y, Pearce JM, McGregor A (2018) The response strategy and the place strategy in a plus-maze have different sensitivities to devaluation of expected outcome. Hippocampus 28(7):484-96. https://doi.org/10.1002/hipo.22847

Lakens D (2017a) TOSTER: two one-sided tests (TOST) equivalence testing. https://cran.rproject.org/web/packages/TOSTER/citation. html

Lakens D (2017b) Equivalence tests. Soc Psychol Pers Sci 8(4):35562. https://doi.org/10.1177/1948550617697177

Lauzon C, Caffo B (2009) Easy multiplicity control in equivalence testing using two one-sided tests. Am Stat 63(2):147-54. https:// doi.org/10.1198/tast.2009.0029

Levy DJ, Glimcher PW (2012) The root of all value: a neural common currency for choice. Curr Opin Neurobiol Decis Mak 22(6):102738. https://doi.org/10.1016/j.conb.2012.06.001

Maddux J-M, Kerfoot EC, Chatterjee S, Holland PC (2007) Dissociation of attention in learning and action: effects of lesions of the amygdala central nucleus, medial prefrontal cortex, and posterior parietal cortex. Behav Neurosci 121(1):63-79. https://doi.org/10. 1037/0735-7044.121.1.63

Marsh B, Kacelnik A (2002) Framing effects and risky decisions in starlings. Proc Natl Acad Sci 99(5):3352-5. https://doi.org/10. 1073/pnas.042491999

McNamara JM, Trimmer PC, Houston AI (2014) Natural selection can favour "irrational behaviour"'. Biol Lett 10(1):20130935. https:// doi.org/10.1098/rsbl.2013.0935
Monteiro T, Vasconcelos M, Kacelnik A (2013) Starlings uphold principles of economic rationality for delay and probability of reward. Proc R Soc B Biol Sci 280(1756):20122386. https://doi.org/10. 1098/rspb.2012.2386

Nachev V, Winter Y (2012) The psychophysics of uneconomical choice: non-linear reward evaluation by a nectar feeder. Anim Cogn 15:393-400. https://doi.org/10.1007/s10071-011-0465-7

Nachev V, Winter Y (2019) Behavioral repeatability and choice performance in wild free-flying nectarivorous bats (Glossophaga commissarisi). Behav Ecol Sociobiol 73(2):24. https://doi.org/ 10.1007/s00265-019-2637-4

Nachev V, Stich KP, Winter Y (2013) Weber's law, the magnitude effect and discrimination of sugar concentrations in nectar-feeding animals edited by Jacob Engelmann. PLoS One 8(9):e74144. https://doi.org/10.1371/journal.pone.0074144

Nachev V, Kai KP, Winter C, Bond A, Kamil A, Winter Y (2017) Cognition-mediated evolution of low-quality floral nectars. Science 355(6320):75-78. https://doi.org/10.1126/science.aah4219

Ojeda A, Murphy RA, Kacelnik A (2018) Paradoxical choice in rats: subjective valuation and mechanism of choice. Behav Process 152(July):73-80. https://doi.org/10.1016/j.beproc.2018.03.024

Packard MG, McGaugh JL (1996) Inactivation of hippocampus or caudate nucleus with lidocaine differentially affects expression of place and response learning. Neurobiol Learn Mem 65(1):65-72. https://doi.org/10.1006/nlme.1996.0007

Pitz GF, Sachs NJ (1984) Judgment and decision: theory and application. Annu Rev Psychol 35(1):139-64. https://doi.org/10.1146/ annurev.ps.35.020184.001035

R Core Team (2020) R: a language and environment for statistical computing. R Foundation for Statistical Computing, Vienna, Austria. http://www.R-project.org

Reid CR, Garnier S, Beekman M, Latty T (2015) Paradoxical choice in rats: subjective valuation and mechanism of choice. Behav Process 152(July):73-80. https://doi.org/10.1016/j.beproc.2018. 03.024 (quantitative and comparative analyses of behavior)

Rieskamp J, Busemeyer JR, Mellers BA (2006) Extending the bounds of rationality: evidence and theories of preferential choice. J Econ Lit 44(3):631-61. https://doi.org/10.1257/jel.44.3.631

Rivalan M, Winter Y, Nachev V (2017) Principles of economic rationality in mice. Sci Rep 7(1):17441. https://doi.org/10.1038/ s41598-017-17747-7

Rosenström T, Wiesner K, Houston AI (2016) Scalar utility theory and proportional processing: what does it actually imply? J Theor Biol 404(September):222-35. https://doi.org/10.1016/j.jtbi.2016. 06.003

Scarpi D (2011) The impact of phantom decoys on choices in cats. Anim Cogn 14(1):127-36. https://doi.org/10.1007/ s10071-010-0350-9

Schuck-Paim C, Pompilio L, Kacelnik A (2004) State-dependent decisions cause apparent violations of rationality in animal choice. PLoS Biol 2(12):e402. https://doi.org/10.1371/journal.pbio.00204 02

Shafir S (1994) Intransitivity of preferences in honey bees: support for "comparative evaluation of foraging options". Anim Behav 48(1):55-67. https://doi.org/10.1006/anbe.1994.1211

Shafir S, Yehonatan L (2014) Comparative evaluations of reward dimensions in honey bees: evidence from two-alternative forced choice proboscis-extension conditioning. Anim Cogn 17(3):63344. https://doi.org/10.1007/s10071-013-0694-z

Shafir S, Waite T, Smith B (2002) Context-dependent violations of rational choice in honeybees (Apis Mellifera) and gray jays (Perisoreus Canadensis). Behav Ecol Sociobiol 51(2):180-87. https:// doi.org/10.1007/s00265-001-0420-8

Sih A, Del Giudice M (2012) Linking behavioural syndromes and cognition: a behavioural ecology perspective. Philos Trans R Soc B 
Biol Sci 367(1603):2762-72. https://doi.org/10.1098/rstb.2012. 0216

Trimmer PC (2013) Optimal behaviour can violate the principle of regularity. Proc R Soc Lond B Biol Sci 280(1763):20130858. https://doi.org/10.1098/rspb.2013.0858

Tversky A (1969) Intransitivity of preferences. Psychol Rev 76(1):3148. https://doi.org/10.1037/h0026750

Tversky A, Kahneman D (1974) Judgment under uncertainty: heuristics and biases. Science 185(4157):1124-31. https://doi.org/10.1126/ science.185.4157.1124

Vora SR, Camci ED, Cox TC (2016) Postnatal ontogeny of the cranial base and craniofacial skeleton in male C57BL/6J mice: a reference standard for quantitative analysis. Front Physiol. https://doi.org/ 10.3389/fphys.2015.00417

Zocchi D, Wennemuth G, Oka Y (2017) The cellular mechanism for water detection in the mammalian taste system. Nat Neurosci 20(7):927-33. https://doi.org/10.1038/nn.4575

Publisher's Note Springer Nature remains neutral with regard to jurisdictional claims in published maps and institutional affiliations. 